\title{
La experiencia vivida de la emergencia obstétrica: un estudio fenomenológico con mujeres mexicanas'
}

\section{The lived experience of the obstetric emergency: \\ a phenomenological study with Mexican women}

\author{
Yesica Yolanda Rangel-Flores ${ }^{\text {a }}$ \\ (D) https://orcid.org/0000-0001-5673-6891 \\ E-mail: yesica.rangelळuaslp.mx \\ Diana Topacio Rincón-Zúñiga ${ }^{a}$ \\ (D) https://orcid.org/0000-0002-7783-2687 \\ E-mail: topacio.rinconळgmail.com \\ Luis Eduardo Hernández-Ibarra ${ }^{a}$ \\ (1D) https://orcid.org/0000-0002-3889-7533 \\ E-mail: eduardo.ibarraळuaslp.mx \\ aUniversidad Autónoma de San Luis Potosí. Facultad de \\ Enfermería y Nutrición. Programa de Maestría en Salud Pública. \\ Universidad Autónoma de San Luis Potosí. San Luis Potosi, \\ S.L.P., México.
}

\section{Resumen}

Este estudio pretende profundizar en la experiencia vivida por mujeres mexicanas en la emergencia obstétrica. Para ello, se realizó un estudio cualitativofenomenológico por medio de entrevistas en profundidad a 15 mujeres sobrevivientes de esta experiencia que se realizaron en el periodo de enero a septiembre de 2017. La información se procesó mediante análisis fenomenológico del discurso, y para su interpretación se organizó en las cuatro dimensiones de la fenomenología de la percepción: cuerpo, tiempo, espacio y relacionabilidad. Las informantes percibieron la complicación de forma temprana, sin embargo, postergaron la atención institucional hasta que se hicieron presentes signos objetivos de complicación; la llegada puntual a los servicios de salud no implicó, desde su percepción, la certeza de manejo oportuno y adecuado, además se reportó un insuficiente apoyo de las redes formales e informales para el traslado y la atención especializada. Es necesario que sociedad y gobierno articulen estrategias que empoderen a las mujeres para la atención a las situaciones de emergencia obstétrica, acciones que promuevan su autocuidado pero garanticen también el apoyo pronto y expedito de sus redes y una atención profesional de calidad.

Palabras clave: Emergencia; Obstetricia; Experiencia; Fenomenología; Investigación Cualitativa.

\section{Correspondencia}

Yesica Yolanda Rangel-Flores. Av. Niño Artillero, 130. Zona Universitaria. Ext. 5083. San Luis Potosí, S.L.P. México. CEP 78240.

Estudio realizado con recursos del Programa para el Desarrollo Profesional Docente, para el Tipo Superior (PRODEP), a través de la beca UASLP-PTC-578 otorgada a quien aparece como primera autora. 
This article aims to explore the experience of Mexican women in the obstetric emergency. This qualitative-phenomenological study was conducted with data collected by means of indepth interviews with 15 women survivors of this experience, performed from January to September 2017. Obtained data were processed through discourse analysis and interpreted based on the four dimensions of the phenomenology of perception, namely: body, time, space, and relationality. Despite perceiving the complications at early stages, interviewees waited for objective symptoms before seeking for healthcare. According to them, timely treatment seeking did not imply an adequate management. Moreover, these women also reported insufficient support from both formal and informal networks in the provision of specialized treatment. Society and government must articulate strategies that empower women for the attention to obstetric emergencies, besides implementing actions that promote their self-care while guaranteeing timely and specialized care. Keywords: Emergency; Obstetrics; Experience; Phenomenology; Qualitative Research.
La muerte materna (MM) continúa siendo un grave problema en la mayor parte de los países subdesarrollados, los que contribuyen de manera importante en las 830 muertes que se calculan diariamente en el mundo. América Latina es una de las regiones que, junto con África Subsarihana y Asia, representa una región álgida para la muerte materna, tan solo en este continente ocurrieron en 2015, 60 defunciones maternas por cada cien mil nacimientos, lo que se tradujo en una reducción de apenas el 52\% para el periodo 1990-2015 (CELADE, 2015).

En México la reducción de la MM para el periodo $1990-2013$ fue de un $26,8 \%$ apenas, casi la mitad de lo que descendió en toda América Latina, posicionándolo en el décimo lugar entre los países de este continente y por debajo del promedio logrado por el mismo (Kassebaum et al., 2014). En este país como en la mayor parte del mundo, la mayoría de las muertes maternas se asocian a trastornos hipertensivos del embarazo, hemorragia (por parto prolongado u obstruido, ruptura uterina y embarazo ectópico), abortos y sepsis. Las situaciones antes descritas se clasifican como emergencias obstétricas, entendiéndolas como "estados nosológicos que ponen en peligro la vida de la mujer durante la etapa grávido-puerperal y/o al producto de la gestación que requiere de atención médica y/o quirúrgica inmediata por personal médico calificado" (Centro Nacional de Equidad de Género y Salud Reproductiva, 2016).

La relevancia de la identificación oportuna por parte de las mujeres, la búsqueda del traslado y el tratamiento oportuno cobra relevancia cuando se ha documentado que el tiempo promedio para que se produzca la muerte posterior al desencadenamiento de una emergencia obstétrica es de dos horas para el caso de una hemorragia, dos días en promedio para la eclampsia y el parto obstruido, y hasta seis días en el caso de algún proceso infeccioso (Ouédraogo, 2010).

En la revisión documental de los estudios que se han realizado en torno a las emergencias obstétricas, se identificó la prevalencia de estudios que se profundizan en el desempeño del personal sanitario en el momento del evento, dejando de lado el documentar las disyuntivas que enfrentan las mujeres para identificar el problema y buscar el traslado. 
Los escasos estudios que exploraron las perspectivas de las mujeres se han centrado en identificar su nivel de conocimiento respecto a las señales de alarma (García; Montañez, 2012), su percepción sobre la calidad de la atención médica recibida (Mohd et al., 2017) y los obstáculos para acceder a la atención profesional (Hussein et al., 2016). Otros estudios por su parte, aunque parten de trabajar con mujeres que han vivido una emergencia obstétrica, centran su interés en explorar el impacto que esta experiencia trajo a su vida posterior al evento y no en la conciencia que como tal desarrollan durante la emergencia (Madeline, 2016; Elmir, 2014).

No obstante, únicamente un estudio tuvo como objetivo profundizar en la experiencia de la emergencia obstétrica y reportó que esta experiencia es vivida como un suceso traumático y conflictivo, cuyos alcances impactan en la demanda, confianza y los miedos hacia los profesionales y el sistema de salud en general (Szulik; Szwarc, 2015). Cabe señalar que la investigación no se trataba de un acercamiento fenomenológico, sino de una etnografía.

A partir de la revisión de las investigaciones realizadas sobre el tema, se consideró pertinente explorar la experiencia de la emergencia obstétrica desde la fenomenología, considerando que este enfoque permite develar cómo las mujeres viven los fenómenos desde la significación que hacen de los procesos, en su consciencia y a partir de una perspectiva que, aunque se considera individual, se conoce fundamentada en maneras de ver y habitar el mundo que encuentran sentido dentro de los colectivos en que estas personas se han desarrollado (Berger; Luckmann, 2005).

Ante esto, el objetivo de esta investigación fue profundizar en la experiencia vivida por las mujeres de México en la emergencia obstétrica, apostando que lo que ellas tengan que decir sobre su propia experiencia y desde su subjetividad contribuirá a construir políticas públicas en salud sensibles a distintas realidades.

\section{Métodos y procedimientos}

Este es un estudio cualitativo desde la fenomenología de la percepción propuesta por Merleau Ponty, toda vez que lo que interesaba era comprender en su complejidad la experiencia vivida, interpretando los significados que subyacen a la manera de reconstruir desde la propia subjetividad dicho evento.

La información se recolectó durante el periodo de enero a septiembre de 2017 , con 15 mujeres residentes en la región centro norte de México. Se decidió trabajar con mujeres de esta región porque existen distintas condiciones de desarrollo humano y bienestar social con el potencial de determinar experiencias emocionales diversas al momento de la emergencia obstétrica, lo que contribuye a la validez externa del estudio.

Las potenciales participantes fueron identificadas a partir de los registros de servicios de ginecobstetricia de hospitales públicos de la región. Tras una revisión minuciosa y sistemática de los expedientes clínicos, se seleccionó aquellas que hubiesen cursado por una emergencia obstétrica en los últimos tres años, sin que hubieran vivido derivado de ello una muerte perinatal. La decisión de no invitar a mujeres cuyos hijos hubiesen muerto obedeció principalmente a consideraciones éticas, puesto que se supuso que quienes hubieran vivido una pérdida perinatal tendrían mayor vulnerabilidad emocional, lo que significaría en sí mismo un riesgo para su seguridad psicológica.

Una vez identificadas, se les contactó por llamadas telefónicas para acordar un primer encuentro con las entrevistadoras. En este primer encuentro, se explicó la naturaleza y alcance del estudio, y se conversó con ellas para confirmar que lo documentado en el expediente clínico coincidía con su experiencia. En el marco antes señalado, se implementó la estrategia de muestreo propositivo (Mendieta, 2015), toda vez que se invitó a participar a mujeres que hubiesen vivido en profundidad el fenómeno de la emergencia obstétrica para que recordaran el evento y quisieran platicar de ello.

En un segundo encuentro se obtuvo el consentimiento informado y se realizó la entrevista inicial. La información se recolectó mediante entrevista fenomenológica cuya pregunta central que desencadenó el relato fue: “PPodría relatarnos cómo vivió el día en que su hijo(a) nació?”. Ambas entrevistadoras procuraron que las mujeres tuviesen libertad para construir sus propios relatos 
y la única intervención que realizaron consistía en cuidar la narrativa cronológica de los relatos, con el fin de evitar lagunas de información que pudieran traducirse en ausencias que complicaran la comprensión clara de la experiencia por parte del equipo de investigación.

Las entrevistas fueron realizadas por dos investigadoras del equipo de investigación. La decisión de que fuera mujeres quienes entrevistaban a las sobrevivientes tuvo en cuenta que, dado que los procesos reproductivos son contemplados también como sexuales, ser entrevistada por un varón podía mermar la riqueza de los relatos que las mujeres construyeran.

El número de entrevistas se determinó en función del criterio de saturación de las experiencia, y se dejó de entrevistarlas una vez que comenzaban a construir narrativas redundantes sobre su experiencia. Las entrevistas tuvieron una duración de aproximadamente 90 minutos, y una sola entrevista resultó suficiente para profundizar en la experiencia de la emergencia obstétrica.

Las entrevistas fueron audiograbadas bajo previo consentimiento informado, posteriormente fueron transcritas por las investigadoras que realizaron las entrevistas. Una vez hecho esto, los audios fueron eliminados para garantizar la protección de los datos proporcionados por las informantes. Sobre las entrevistas transcritas se aplicó el análisis fenomenológico-interpretativo, siguiendo las etapas: (1) Transcripción, (2) Elaboración de Unidades de significado general, (3) Elaboración de Unidades de significado relevante para el tema de la investigación, y (4) Verificación de las unidades de significado relevante.

Para el análisis de los relatos, las unidades de análisis se agruparon en cuatro dimensiones que considera la fenomenología: La corporalidad (el cuerpo vivido), la temporalidad (el tiempo vivido), la espacialidad (el espacio vivido), y la relacionabilidad (las relaciones de interacción vividas) (Álvarez et al., 2010).

El estudio cumplió con los criterios éticos y legales que rigen la investigación tanto en el ámbito internacional como en el nacional, particularmente en la Declaración de Helsinki y lo que marca en materia de investigación la Ley General de Salud de México. El estudio se clasificó de riesgo mínimo, puesto que se anticipó que las participantes podían desencadenar crisis emocionales por rememorar un episodio doloroso de su vida. En este sentido, se planteó de antemano suspender la entrevista en caso de crisis emocional, brindar contención y hacer acompañamiento psicológico. Pero no se presentaron situaciones de crisis. El protocolo fue aprobado y monitoreado por el Comité de Ética en Investigación de los Servicios de Salud de San Luis Potosí, con registro SLP/oo2/2017.

\section{Resultados}

\section{Caracterización sociodemográfica}

Se trabajó con 15 mujeres, con un promedio de edad de 22,9 años, una mínima de 16, máxima de 39 y moda de 18 ; el $86,6 \%$ se dedicaba a las labores de casa, el 6,6\% era empleada y el 6,6\% estudiaba. La mayoría había cursado la secundaria completa $(46,6 \%)$ y la primaria completa $(33,3 \%)$, no había mujeres profesionistas. El $80 \%$ se encontraba dentro de una relación de unión libre y matrimonio al momento en que se presentó la emergencia obstétrica, y el $20 \%$ se refirió como madre sola.

\section{Perfiles obstétricos y caracterización del evento de emergencia obstétrica}

El evento de emergencia obstétrica se presentó cuando la mayoría cursaba su primera gestación $(66,6 \%)$, seguido por secundigestas $(26,6 \%)$ y había un caso de cuarta gesta. En lo que respecta a la edad gestacional, el $40 \%$ de las mujeres vivió la experiencia entre las 38 y las 39.6 semanas de gestación (SDG), el 26,6\% entre las 32 y 34 SDG, y el $20 \%$ posterior a las 40 SDG.

Respecto a la situación que derivó en la emergencia obstétrica, el 93,3\% ( $\mathrm{N=14}$ ) tuvo un diagnóstico de ingreso asociado con trastornos hipertensivos del embarazo, de las cuales, el 21,4\% $(\mathrm{N}=3)$ ingresaron con eclampsia en desarrollo y el $14,2 \%(\mathrm{~N}=2)$ con síndrome de Hellp. La otra participante ingresó con cuadro de septicemia, posterior a resolución de parto por cesárea y antecedente de diabetes mellitus. 


\section{Dimensión I: la emergencia en la corporalidad}

Cuando el cuerpo que se siente mal no es suficiente, la necesaria validación del malestar desde los otros

Las participantes coincidieron en haber intuido de manera temprana -el día que se presentó la emergencia obstérica- que algo no andaba bien en sus cuerpos. Sin embargo, todas consideraron que era prematuro e innecesario solicitar la ayuda de sus redes familiares o profesionales, dado que los datos de complicación obstétrica se quedaban en el plano subjetivo (molestias) y no eran evidentes, por lo que no podían ser sujetos de comprobación por otras personas (familiares o profesionales). En función de lo anterior, la mayor parte de las mujeres prefirió continuar con sus actividades cotidianas, esperando a que se manifestaran signos que pudieran ser observables por sus familiares y justificaran la necesidad de atención especializada. Algunos de los signos que se hicieron evidentes fueron la imposibilidad para mantener la marcha, edema grave, vómito, dolor incapacitante, entre otros.

Romina por ejemplo refirió sentirse aliviada cuando finalmente en la madrugada desencadenó el vómito, cefalea intensa y dificultades para mantener una marcha autonóma; tras haber pasado toda una tarde con dolor epigástrico y la intuición de que esto no era normal. Pensó que, con todos estos datos objetivos de complicación, sus familiares no dudarían en acercarle al hospital y que en el centro sanitario no postergarían su atención, puesto que se veía realmente mal:

Yo había estado con el dolor fuerte desde la tarde, aquí [señala el epigastrio]. Pensaba que podía ser por el bebé, pero no estaba segura, no sabía si, sí, estaba mal, no me veía tan mal, hasta que en la noche empecé con mucho vómito, el dolor de cabeza, el mareo de no poder caminar. Eso ya fue en la madrugada, me asusté, pero fue cuando ya supimos que estaba mal, y como me dijeron [esposo y suegra] que teníamos que irnos al hospital, que tenía que ir a que me atendieran ipero ya!. (Romina)

Lastimosamente, no todas las mujeres narraron haber mantenido la consciencia cuando estos datos de gravedad se hicieron presentes, tal fue el caso de
Micaela, quien no se consideró lo suficientemente mal, hasta que la aparición de las convulsiones:

Me sentía mal, la cabeza me dolía, sentía que no veía bien, estaba mareada, pensé ir al hospital, pero dije "a lo mejor durmiendo se me pasa", es que no me veía mal, mal, no. Yo podía andar todavía, me quedé dormida hasta que como a las tres de la mañana empecé a convulsionar, mi hermana dormía en el mismo cuarto, eso fue lo que hizo que ella saliera a decir, ya cuando me desperté estaba en el hospital. (Micaela)

\section{Dimensión II: el tiempo en la emergencia}

La espera paciente de la evolución de la complicación

Varias participantes refirieron saberse diagnosticadas con un trastorno hipertensivo del embarazo antes de la emergencia obstétrica, incluso, había solicitado atención profesional en los servicios de urgencia días antes del evento. También narraron haber vivido la experiencia de ser regresadas a su domicilios en más de una ocasión, bajo el argumento de que no había "datos" suficientes para su ingreso:

Yo sabía que andaba con la presión alta, entonces fui un viernes, me checó y me dijo que regresara el sábado y que depende de lo que vieran me iba a quedar. Y fue ahí cuando, fui el sábado y fue cuando ya se me subió ahora sí bastante la presión, fue entonces cuando ya me metieron de urgencia para la operación. (Ángela)

Se identificó, entonces, que el tiempo que marca la evolución en la experiencia y resolución de la emergencia obstétrica no corresponde con los procesos fisiológicos que acontecen en sus cuerpos o en la toma de decisiones que las mujeres hacen respecto a en qué momento buscar la atención especializada, sino que depende inexorablemente de los tiempos institucionales y el juicio que elabore el personal médico sobre sus casos.

Como los últimos dos meses estuve yendo a cada rato, me sentía mal, ya me habían internado una vez, esa vez fue por la presión, a la segunda fue por lo mismo, por la presión alta pero me dieron que no 
estaba tan alta, pero yo me sentía deberás mal, no aguantaba la cabeza, veía mal, pero que dicen que no, que ya se controló y me regresan a mi casa. (Rubí)

Otra situación fue la subestimación que hacen las propias mujeres sobre el escaso tiempo de que dispone el personal especializado para garantizar la vida de ellas y sus hijos, una vez que se desencadena una emergencia obstétrica. Varias retrasaron la búsqueda de la atención, por anteponer el cumplimiento de una serie de funciones sociales asociadas al género, principalmente asociadas a la crianza y el cuidado de otros, así como lo narra Catalina:

Me empecé a sentir mal desde que me levanté, me sentía con un dolor muy fuerte de cabeza, pero me daba y como que se me quitaba, pero no era muy fuerte, entonces así seguí. Mis hermanas trabajan y yo les cuidaba sus niños y, pues ya me los trajeron, y yo como si nada pues el dolor me daba y se iba, me dejaron a los niños y fue hasta que regresaron por ellos que le dije a mi mamá que teníamos que ir al hospital. (Catalina)

Sandra por ejemplo desarrolló septicemia posterior a la cesárea y, aunque los datos de la complicación se hicieron evidentes de manera temprana, ella retrasó lo más que pudo la salida al hospital, con la finalidad de no ausentarse del cuidado de su hijo:

La temperatura nada más no se me bajaba, me metía a bañar y no bajaba de 39 y se subía hasta 40 , ya iban y me ponía lo que era toallas mojadas en la frente y en el estómago para poderme bajar la temperatura, me querían llevar al hospital, pero yo le pensaba porque iba a dejar al niño y no le iba a poder dar de comer... aquí estaba él [esposo] y mis hijas que ya están grandes, pero yo era la que lo cuidadaba. (Sandra)

Lo anterior se complejiza aún más cuando se está embarazada y se conoce aún lejana la fecha estimada para el parto. El miedo a que el bebé nazca antes de poder mantener la vida por sí mismo fue una de las razones que retrasó la salida hacia las instituciones sanitarias, refiriendo miedo de ser operadas y tener hijos prematuros.

\section{Dimensión III: espacio y emergencia}

Los hospitales como espacios no siempre resolutivos

El lugar en que ocurre la emergencia obstétrica no parece ser una cuestión determinante respecto al manejo del evento adverso. Alejandra que inició con convulsiones en una cancha comunitaria de futbol se encontró con una respuesta comunitaria asertiva, en la que procuró el traslado ágil hasta un hospital de segundo nivel de atención, a diferencia de ella, Karla cuyo evento de emergencia obstétrica se identificó mientras asistía a una consulta de control prenatal, presenció una serie de vicisitudes que retrasaron la referencia y el traslado entre el primer y un segundo nivel de atención.

El caso de Karla resultó particularmente relevante, toda vez que se esperaría que el traslado fuera mucho más ágil entre servicios de salud de primer y segundo nivel de atención en función de la existencia de protocolos de referencia y contrarreferencia. Esto no ocurrió así por lo menos en su experiencia:

Ese día yo fui a consulta, no porque me sintiera mal, nomás que me tocaba consulta. El Doctor que me atendió me regañó y me dijo que porque no había ido antes que traía la presión muy alta, me mandó al hospital pero no encontraba mi expediente, tuvo que hacer otra vez todo el papeleo, pidió que me acompañara una trabajadora social hasta el hospital, pero nos tardamos mucho, porque a ella no le querían prestar la camioneta porque la iban a ocupar en otra cosa del mismo centro de salud. (Karla)

\section{Dimensión IV: las relaciones en la emergencia}

El apoyo insuficiente en las redes formales e informales

Se identificó un insuficiente apoyo tanto de las redes formales como informales. Respecto a las primeras, se documentó la experiencia de violencia de género como una condición con potencial para influir negativamente en los resultados de la emergencia obstétrica. Este fue el caso de Bere quien buscó las estrategias para salir de la casa de su pareja y poder acercarse a la de sus padres, donde 
contemplaba una red más sólida para garantizar su bienestar y el de su hijo:

Yo estaba con él, en la casa, ahí empezaron a darme las primeras molestias, me dolía la cabeza, me sentía que no veía bien y con mucho cansancio en la espalda, pero haga de cuenta que yo sabía que él no me iba a llevar. Entonces hice el plan con maña de yo pelearme con él para venirme aquí [casa de la madre], porque yo sabía que necesitaba que alguien me llevara si me sentía más mal. (Berenice)

Pero esta experiencia de sentirse temerosas, inseguras y rodeadas de personas con una capacidad insuficiente para garantizar su seguridad también fue vivida dentro de servicios sanitarios de primer nivel de atención:

No encontraban mi expediente, él [el doctor] se puso [a] hacer un expediente que porque según yo no tenía, pero me estuvo regañando, como si yo tuviera la culpa de que no encontraran el expediente, me quitó la cartilla que traía y me hizo otra, le dije que me diera la mía porque me la iban a pedir en el hospital, no me la quiso dar... (Karla)

\section{Consideraciones finales}

En este estudio pudo documentarse que las mujeres no cuentan con las competencias necesarias (de conocimiento, de actitud y valorativas) para identificar y actuar frente a las señales de alarma iniciales y en dicho tenor subjetivas. En lo que respecta a las competencias de conocimiento, se identificó que las informantes percibieron las molestias, sin embargo no las problematizaron asociadas con estados patológicos de la gestación. Mengole y Lannacone (2009) han señalado con antelación que a menor edad las mujeres presentan no solo una falta de conocimiento sobre las señales de alarma, sino un desconocimiento en general sobre sexualidad y reproducción.

En lo que respecta a las competencias de actitud en relación a en qué momento y a quién comunicar la identificación de estas señales de alarma, se encontró que incluso una vez que se identifica que algo está fuera de la normalidad, las mujeres no siempre cuentan con las redes para asegurar el traslado pronto y expedito. Además, muestran resistencia para acercarse de manera temprana a los servicios de salud. Mengole y Lannacone (2009) también han documentado la existencia de una actitud negativa en relación al acercamiento con los servicios de salud para solicitar atención.

Finalmente, pero no menos importante, en cuanto a las competencias valorativas, se identificó que las mujeres no se asumieron derivado de su experiencia de emergencia obstétrica, cercanas a la muerte, lo cual definitivamente dictó el tenor de la evolución que dejaron avanzar en sus estados de morbilidad materna extrema. Además, se documentó que prevalece una desconfianza y falta de certeza sobre la eficacia con la que podían ser atendidas dentro de los servicios de salud.

En lo que respecta a la experiencia de la emergencia vivida desde la corporalidad se identificó que existe una distancia simbólica dentro del imaginario desde el cual estas mujeres significan el cuerpo durante el evento de la emergencia obstétrica, es decir, no se significa igual el cuerpo "que se siente enfermo" que el cuerpo "que se nota enfermo". Las razones que obligan a las mujeres a esperar pacientes la manifestación de signos objetivos y en dicho sentido verificables obedece, como hemos dado cuenta en las narrativas de las mujeres, a la necesidad de tener certezas sobre el apoyo que recibirán de sus redes de apoyo familiares e institucionales. Tal y como han documentado Modh et al. (2017) en un estudio realizado en Malasia, el afrontamiento de las redes de apoyo familiar para hacer el traslado oportuno suele ser ineficaz cuando no son suficientemente evidentes las señales de alarma.

La carencia de experiencias previas contra las cuales validar la sintomatología subjetiva asociada al momento inicial de la emergencia obstétrica puede ser el obstáculo principal para identificar la complicación inicial e incluso la confianza de acercarse a los servicios de salud pertinentes para ser auxiliada. García y Montañez (2012) han señalado al igual que nosotros que uno de los determinantes de mayor peso en la identificación de señales de alarma es el hecho de haber estado embarazadas de 
manera previa, dado que el discurso médico recibido sobre los cambios fisiológicos y patológicos se han evaluado en el marco de una experiencia vivencial.

Por otra parte, consideramos relevante destacar que en nuestro estudio prevaleció la presencia de informantes cuyas edades se adscriben a la etapa de juventud, lo que respalda un hallazgo que con antelación ha sido señalado en estudios epidemiológicos sobre morbilidad materna extrema (Oliveira et al., 2014). En nuestro estudio igual que en los de los autores antes señalados, no figuraron mujeres mayores de 40 años, lo que explicamos al tenor de lo dicho antes por Oliveira et al. (2014) respecto a que "las mujeres en límites etários mayores tienen hasta un $25 \%$ más probabilidades de morir", lo que hace probable que mujeres de mayor edad no figuren como sobrevivientes, sino que hayan sido casos de muerte materna.

Las razones que desalentaron a las mujeres a comunicar las molestias iniciales fue el temor de que estas fueran puestas en duda por sus familiares o el personal sanitario, ya que se trataba de síntomas (en dicho sentido no verificables). El miedo a comunicar la existencia de una complicación de la que no están ciertas, así como la inseguridad en el apoyo de los terceros (familiares y personal sanitario), ha sido referido por Perón y Da Silva (2011) como una situación que desalienta la comunicación oportuna de las señales de alarma obstétrica.

La falta de seguridad de las mujeres sobre su capacidad de escuchar, interpretar y relacionarse con sus propios cuerpos ha sido asociada por Bedoya (2019) como consecuencia de la compleja medicalización que sobre los cuerpos femeninos, más específicamente sobre los procesos reproductivos, se ha hecho y fortalecido a lo largo del último siglo. Con la tecnificación del abordaje de los cuerpos desde la medicina occidentalizada, se ha centrado la atención en la medición de lo objetivo, invisibilizando lo subjetivo, particularmente cuando se trata de la subjetividad de las mujeres, un género al que se ha construido en el imaginario colectivo -en contraparte de lo masculino- con tendencia a magnificar en sus estados de ánimo a través de personalidades histriónicas (APA, 2013).

Ahora, si bien la medicalización, tecnificación y mecanización asistencial que caracteriza la atención de los procesos reproductivos de las mujeres dentro de las instituciones posmodernas no solo no ha podido demostrar una relación directa con la reducción de la morbimortalidad materno-infantil (Hernández; Echeverría; Gomariz, 2017), sino que ha impulsado que las mujeres aprendan a desconfiar de sus propios saberes e intuiciones para interpretar y validar las señales de sus cuerpos, convenciéndolas que son otros quienes pueden y deben interpretarlos, son esos otros quienes prescriben incluso como deben sentirse en la gestación, procurando por supuesto que su experiencia encuadre con lo que está escrito en libros que, desde una ciencia positivista, encuentra innecesario incorporar la experiencia subjetiva de las mujeres que viven estos procesos. Desapropiar a las mujeres de su subjetividad para entender el propio cuerpo, representa de hecho uno de los más importantes problemas en el plano de la bioética, puesto que las coloca en un estado de subordinación en relación con sus propios cuerpos, restándoles autonomía para decidir y hacer con ellos (Urrea, 2012).

La perspectiva médica occidentalizada ha contribuido también a fomentar el desarrollo de un imaginario social que representa los procesos reproductivos a manera de procesos patológicos y/o de riesgo. Posicionar la gestación y el parto desde una perspectiva que patologiza y justifica un protocolizado intervencionismo ha generado que las mujeres que cursan dichos procesos los contemplen como situaciones que "es normal" el malestar, consecuentemente, normalizan la sintomatología incipiente de la emergencia obstétrica, que en un primer momento no son perceptibles para los demás porque no son incapacitantes. Godoy et al. (2009) han abordado anteriormente como esta visión occidentalizada del embarazo y el parto patologiza estas etapas y contribuye a invisibilizar señales iniciales de alarma, y en consecuencia retrasa la búsqueda de atención especializada.

En lo que respecta a la segunda dimensión, que es la experiencia vivida desde el tiempo, contrario a lo que han reportado otros estudios (Aguiar; Tanaka, 2016; Szúlik; Szwarc, 2015), ninguna de las participantes en este estudio refirió haberse percibido en una situación cercana a la muerte, lo que puede explicarse en función de lo que 
Godoy et al. (2009) han señalado respecto a que en las mujeres prevalece una falta de conocimiento que no les posibilita dilucidar los potenciales y graves alcances del evento de la emergencia obstétrica.

La mayor parte de las informantes -incluso las que se sabían diagnosticadas con un trastorno hipertensivo del embarazo- narraron haber esperado pacientes el agravamiento de las molestias incipientes. El sentido de esta espera se debió en muchos de los casos al miedo de que estas molestias fueran puestas en duda por familiares o personal de salud, una vez comunicadas. Verse "lo suficientemente mal", desde su imaginario, reduce la probabilidad de un rechazado para el ingreso hospitalario, aspecto que era importante para ellas, particularmente para las que habían sido rechazadas para su ingreso en ocasiones anteriores.

El hallazgo anterior resulta relevante, pues pone en evidencia la razón por la que las mujeres no se acercan de manera temprana a los servicios de salud, no se explica inexorablemente en una resistencia personal para acercarse al personal sanitario, como lo ha señalado Mengole y Lannacone (2009), sino por el contrario, las mujeres han buscado la atención especializada con antelación, dado que su experiencia se ha significado negativa, en el momento de la emergencia obstétrica se torna en una decisión compleja acercarse a los servicios de salud. La existencia de sistemas de referencia y contrarreferencia ha sido un tema polémico en la investigación sobre políticas de salud que se ha desarrollado en México. Collado y Sánchez (2012) han documentado como el sistema de referencia y contrarreferencia en el ámbito de la atención obstétrica es inexistente y no solo eso, sino la ocurrencia de un fenómeno de multirrechazo hospitalario, que vulnera el derecho a la salud en las mujeres y sus hijos.

Estas prácticas de multirrechazo institucional pueden derivar en muertes maternas, pero incluso el que no las haya no significa que esta práctica no tenga un impacto negativo en la salud materna. Collado y Sánchez (2015) han dado cuenta igual que hemos hecho en este estudio que la falta de apego a los lineamientos de referencia y contrarreferencia deriva en un retraso en la llegada de las mujeres con condiciones de morbilidad materna extrema a las instituciones de segundo nivel de atención, principalmente porque en el marco de estas experiencias previas, las mujeres pierden confianza y credibilidad en las acciones y los actores institucionales. Agregaremos a lo anterior que estas prácticas de multirrechazo las enseñan a dudar de su agencia para identificar de manera fehaciente las señales de alarma.

El tiempo se instaura entonces como una variable que no está determinada por la toma de decisión o la autonomía de las mujeres cuya vida está en riesgo, sino que se sitúa en el escenario de las instituciones, corporaciones con una dinámica burocratizada y otro sentido del tiempo. Lo anterior resulta relevante en función de la afirmación que hace Merleau-Ponty respecto que el tiempo no existe en las cosas, sino en las relaciones con las cosas (Hirose et al. 2015). Los tiempos que se juegan entre que se identifica y se resuelve una emergencia obstétrica no dependen de forma absoluta de la agencia que tienen las mujeres y sus familias para llegar hasta una institución hospitalaria, tampoco de la infraestructura y equipamiento que dicha institución tenga, si bien les incluye no debe dejarse de lado el complejo cruce entre las (inter)subjetividades de las mujeres, sus familiares y los actores de salud; cada uno de estos actores, desde su propia subjetividad, determinará que tan urgente es la intervención y el costo que se asumirá de no actuar de manera inmediata.

Pero la ausencia de certeza sobre la gravedad de su condición no es lo único que retrasa la salida de las mujeres de sus casas hacia las instituciones de salud, existe también el cumplimiento de una serie de tareas domésticas derivadas de su rol como amas de casa y madres. En las mujeres que no eran primigestas, el cuidado de otros hijos fue una de las razones por las que no acudieron con premura a revisión obstétrica, lo cual es "natural" en un contexto como el mexicano, en el que la desigualdad de género es resultado y, a su vez, origen de la ausencia de paternidades participativas en la crianza (López, 2017).

La tendencia de las mujeres a devaluar la importancia de su propio bienestar en función de salvaguardar el de sus hijos se hace evidente cuando narran que una de las razones que retrasó su llegada al hospital fue el deseo de que sus embarazos no 
fueran interrumpidos, pues pensaban la prematurez inexorablemente incompatible con la sobrevivencia. Reis et al. (2016) han señalado con antelación como el miedo de la prematuridad posible se asocia con una mayor angustia y sufrimiento al momento de la experiencia y, con ello, entorpece la toma de decisiones para solicitar ayuda.

La tercera dimensión que incorpora la fenomenología es el espacio; para ello, se documentó como a través de las experiencias las sobrevivientes posicionan en su consciencia a las instituciones sanitarias dentro de un plano no resolutivo.

El lugar donde se desencadena la emergencia obstétrica no representa -desde la experiencia de estas mujeres- una variable que diferencie las emociones de angustia, miedo e incertidumbre en las que se enmarca la experiencia; tampoco identifican que el espacio en que se desencadena la situación de emergencia determine la respuesta de apoyo y la resolución del evento. Llamó particularmente la atención el hecho de que se narrara una respuesta comunitaria más pertinente y resolutiva que la mostrada en un centro de atención primaria que cuenta con protocolos para la referencia y contrarreferencia de este tipo de casos, lo cual respalda las afirmaciones de Collado y Sánchez (2015) de que dicho modelo es inexistente en la operatividad.

Encontrar a través de la experiencia vivencial "el sentido" de los lugares es imprescindible para que las personas desarrollen trayectorias de atención que les garanticen certezas y credibilidad en las instituciones. Los resultados de este estudio permiten evidenciar como la experiencia vivida en la emergencia obstétrica contribuye a un fenómeno social que Eyles (1989) ha llamado de “deslugarización” y que se refiere a despojar del sentido socialmente prescrito a un lugar, puesto que en la realidad no funciona para lo que debería hacerlo.

Finalmente, la cuarta dimensión de la fenomenología hace referencia a la relacionabilidad o comunabilidad, entendida esta como las relaciones interpersonales que inciden en la significación de la experiencia, en este caso de la emergencia obstétrica. Lo más significativo en torno a esta dimensión tiene que ver con que no se identificó un apoyo funcional en las redes de apoyo posibles, estas conformadas por la familia y los actores institucionales.
Este estudio documenta que, como ya ha señalado Hirose (2015), no siempre la presencia de una pareja juega como factor protector para la buena resolución de una emergencia obstétrica, principalmente porque nuestro estudio, al igual que el de dicho autor, documenta la existencia de violencia doméstica, lo cual se instituye en una amenaza para la resolución efectiva de la emergencia obstétrica. Mención aparte merece señalar que la violencia doméstica ha sido señalada como una variable asociada a una mayor probabilidad de abortos espontáneos (Biswas et al. 2015). Este hallazgo contradice, o por lo menos exige una posición menos generalista, los argumentos que Haelterman (2003) construye en relación a que la presencia de la pareja juega un papel fundamental en la trayectoria del embarazo y su resolución, atribuyendo "nueve veces más complicaciones en mujeres que no cuentan con pareja durante la gestación, comparadas con quienes la tienen".

En este estudio se identificó, como han hecho también Aguiar y Tanaka (2016), que el desempeño de la red de actores sanitarios no siempre genera certeza y confianza en las mujeres durante este traumático evento, sino que puede potenciar el miedo e incertidumbre que acompaña la experiencia. El miedo a la discriminación y la culpabilización del personal sanitario sobre la situación que les aqueja puede constituirse además en un factor que retrase la búsqueda de ayuda institucional (Rangel; Martinez, 2017).

En resumen, esta investigación ha identificado tres cuestiones asociadas al retraso en la búsqueda de traslado y atención profesional: primero, las mujeres carecen de competencias (de conocimiento, de actitud y valorativas) para identificar las señales de alarma obstétrica; segundo, aquellas que intuyeron que algo anormal estaba sucediendo dudaron ser capaces de intuir que estaban presentando una complicación asociada al embarazo; y tercero, la prevalencia de un imaginario del embarazo como una situación enmarcada en el dolor y la incomodidad, que "normaliza" las molestias iniciales.

La mayoría desestimó el riesgo de muerte asociado a la complicación, principalmente porque al momento del evento no disponían de información 
completa sobre el alcance de la complicación para sus vidas y la de sus hijos, sumado a que la mayor parte era su primer experiencia de gestación y no contaban con una experiencia previa contra la cual validar la (im)pertinencia de las molestias incipientes de la emergencia obstétrica. Finalmente, se identificó que las mujeres no atribuyen una capacidad resolutiva a las instituciones sanitarias y narran procesos de referencia y contrarreferencia inexistentes, de igual forma que el apoyo de las redes formales (instituciones) e informales (familias) es insuficiente. Los hallazgos permiten reflexionar sobre la necesidad de que sociedad y gobierno articulen estrategias que empoderen a las mujeres para la atención a las situaciones de emergencia obstétrica, acciones que promuevan su autocuidado pero garanticen también el apoyo pronto y expedito de sus redes y una atención profesional de calidad.

\section{Referencias}

AGUIAR, C.; TANAKA, A. C. Memórias coletivas de mulheres que vivenciaram o near miss materno: necessidades de saúde e direitos humanos. Cad. Saúde Pública, Rio de Janeiro, v. 32, n. 9, p. 1-13, 2016. DOI: 10.1590/0102-311Хoo161215

ÁLVAREZ, M. et al. Caracterización de la morbilidad materna extremadamente grave. Rev Cubana Hig Epidemiol, La Habana, v. 48, n. 3, p. 310-320, 2010. Disponível em: <http://scielo.sld. cu/pdf/hie/v48n3/hie10310.pdf>. Acesso em: 11 out. 2021

AMERICAN PSYCHIATRIC ASSOCIATION - APA. Diagnostic and statistical manual of mental disorders. 5. ed. Washington, DC: American Psychiatric Association, 2013.

BEDOYA, L.; AGUDELO, A. Relación de las mujeres en embarazo, parto y postparto (EPP) con los servicios de salid según la clase social. Revista Gerencia y Políticas de Salud, Bogotá, DC, v. 18, n. 36, p. 1-12, 2019. DOI: 10.11144/Javeriana.rgsp18-36.rmep

BERGER, P. L.; LUCKMANN, T. La construcción social de la realidad. Buenos Aires: Amorrortu editores, 2005.

BISWAS, A. et al. Intimate partner violence during pregnancy and victim's perception on miscarriage. International Journal of Public Health Research, New York, v. 3, n 2, p. 58-63, 2015. Disponível em: < http://www.openscienceonline. com/journal/archive2?journalId=718\&paper $\mathrm{Id}=1433>$. Acesso em: 11 out. 2021.

CELADE - Centro Latinoamericano y Caribeño de Demografía. Hacia la armonización de las estimaciones de mortalidad materna en América Latina. Serie Población y Desarrollo, Santiago, n. 108, LC/L.3735, 2015. Disponível em: < https://repositorio.cepal. org/bitstream/handle/11362/7143/LCL3735_ es.pdf? sequence=1\&isAllowed=y>. Acesso em: 11 out. 2021.

CENTRO NACIONAL DE EQUIDAD DE GÉNERO Y SALUD REPRODUCTIVA. Triage obstétrico, código mater y equipo de respuesta inmediata obstétrica. Lineamiento técnico. Secretaría de la salud, Ciudad de México, 2016. Disponível em: <https://www.gob.mx/cms/uploads/attachment/ file/131801/web_TriageObstetricoCM.pdf $>$. Acesso em: 11 out. 2021.

COLLADO-PEÑA, S. P.; SÁNCHEZ-BRINGAS, A. Referencia y contrarreferencia o multi-rechazo hospitalario? Un abordaje cualitativo. Revista CONAMED, Ciudad de México, v. 17, n. 1, p. 23-31, 2012. Disponível em: <https://www.medigraphic. com/pdfs/conamed/con-2012/cons121d.pdf >.

Acesso em: 11 out. 2021.

COLLADO-PEÑA, S.; SÁNCHEZ-BRINGAS, A. Dificultades y obstáculos en la atención obstétrica: un estudio de caso. Género y Salud en cifras, Ciudad de México, v. 13, n. 3, p. 25-36, 2015. Disponível em:<https://www.gob.mx/cms/ uploads/attachment/file/245089/13_3Art3.pdf>. Acesso em: 11 out. 2021.

ELMIR, R. Finding meaning in life following emergency postpartum hysterectomy: what doesn't kill us makes us stronger. Journal of Midwifery Womens Health, Silver Spring, v. 59, n. 5, p. 510-515, 2014. DOI: 10.1111/jmwh.12169

EYLES, J. The geography of everyday life. In: GREGORY, D.; WALFORD, R. (Ed.). Horizons in human geography. Totowa: Barnes \& Noble, 1989. p.102-117. 
GARCÍA, J. F.; MONTAÑEZ, M. I. Signos y

síntomas de alarma obstétrica. Acerca del conocimiento que tienen las mujeres. Revista médica del Instituto Mexicano del Seguro Social, Ciudad de México, v. 5o, n. 6, p. 651-657, 2012. Disponível em: <http://new.medigraphic.com/cgi$\mathrm{bin} /$ resumen.cgi? IDARTICULO=41767>.

Acesso em: 11 out. 2021.

GODOY, S. R. et al. Near miss: repercussões e percepção da assistência recebida por mulheres sobreviventes egressas de uma unidade de terapia intensiva. Acta Paul Enferm, São Paulo, v. 22, n. 2, p. 162-168, 2009. DOI: 10.1590/So10321002009000200008

HAELTERMAN, E. et al. Social deprivation and poor access to care as risk factors for severe pre-eclampsia. European Journal of Obstetrics \& Gynecology and Reproductive Biology, Bruselas, v. 111, n. 1, p. 25-32, 2003. DOI: 10.1016/So3012115(03)00161-1

HERNÁNDEZ, J. M.; ECHEVARRÍA, P.; GOMARIZ, M. J. ¿Némesis obstétrica o disminución del riesgo? A debate bioético el abordaje intervencionista en los partos de bajo riesgo. Acta bioethica, Santiago, v. 23, n. 1, p. 161-17o, 2017. DOI: 10.4067/S1726-569X2017000100161

HIROSE, A. et al. Determinants of delays in travelling to an emergency obstetric care facility in Herat, Afghanistan: an analysis of crosssectional survey data and spatial modelling. $B M C$ Pregnancy and Childbirth, Londres, v. 15, n. 14, p. s/n, 2015. DOI: 10.1186/s12884-015-0435-1

HUSSEIN, J. et al. Maternal death and obstetric care audits in Nigeria: a systematic review of barriers and enabling factors in the provision of emergency care. Reproductive health, Londres, v. 13, n. 47, p. 1, 2016. DOI: 10.1186/s12978-016-0158-4

KASSEBAUM, N. et al. Global, regional and national levels and causes of maternal mortality during 1990-2013: a systematic analysis for the Global Burden of Disease Study 2013. The Lancet, Londres, v. 384, n. 9947, p. 980-1004, 2014. DOI: 10.1016/So140-6736(14)6o696-6

LÓPEZ ESTRADA, S. Políticas de cuidado infantil en América Latina: análisis comparado de Chile,
Costa Rica, Uruguay y México. Frontera norte, Tijuana, v. 29, n. 58, p. 25-46, 2017. DOI: 10.17428/ rfn.v29i58.495

MADELEINE, C. Understanding psychological traumatic birth experiences: a literature review. Journal woman and birth, Camberra, v. 29, n. 3, p. 203-207, 2016. DOI: 10.1016/j.wombi.2015.10.009

MENDIETA, G. Informantes y muestreo en investigación cualitativa. Investigaciones Andina, Bogotá, DC, v. 17, n. 30, p. 1148-1150, 2015. Disponível em: <http://www.redalyc.org/ pdf/2390/239035878001.pdf >. Acesso em: 11 out. 2021.

MENGOLE, T.; LANNACONE, J. Factores socioculturales, conocimiento yactitudes de las primigestas adolescentes y no adolescentes frente al embarazo en el Hospital José Agurto Tello Chosica, Lima, Perú, 2009. The Biologist, Lima, v. 8, n. 2, p. 164-178, 2009. Disponível em: <https://dialnet.unirioja.es/servlet/ articulo?codigo $=4004820>$. Acceso em: 20 out. 2021.

MOHD, N.; NIK, N. H.; AB, A.; ZAHARAH, S. The experiences of women with maternal near miss and their perception of quality of care in Kelantan, Malaysia: a qualitative study. $B M C$ Pregnancy and Childbirth, Londres, v. 17, n. 1, p. 1, 2017. DOI: 10.1186/s12884-017-1377-6

OLIVEIRA, F. C.; SURITA, F. G.; PINTO, E. S. J. L.; CECATTI, J. G.; PARPINELLI, M. A.; HADDAD, S. M.; COSTA, M. L.; PACAGNELLA, R. C.; SOUSA, M. H.; SOUZA, J. P. Brazilian Network for Surveillance of Severe Maternal Morbidity Study Group. Severe maternal morbidity and maternal near miss in the extremes of reproductive age: results from a national cross-sectional multicenter study. BMC Pregnancy Childbirth, Reino Unido, v. 14, n. 77, p. 1, 2014.

DOI: 10.1186/1471-2393-14-77

OUÉDRAOGO, I. El tratamiento oportuno de las complicaciones obstétricas como estrategia de reducción de la mortalidad materna, 2010.

Kalu Institute. Disponível em:

$<$ https://kaluinstitute.org/es/el-tratamientooportuno-de-las-complicaciones-obstetricascomo-estrategia-de-reduccion-de-la-mortalidadmaterna-2/>. Acesso em: 11 out. 2021. 
PERON GIAXA, T. E.; DA SILVA, M. L. Miedo e inseguridad de la gestante durante el trabajo de parto como motivos para la demanda de internación precoz. Investigación y Educación en Enfermería, Medellín, v. 29, n. 3, p. 363-369, 2011. Disponível em: <https://www.redalyc.org/pdf/1052/105222406004. pdf $>$. Acesso em: 11 out. 2021.

RANGEL, Y. Y.; MARTÍNEZ, A. G. (In) visibilizaciones de violencia obstétrica en las percepciones de indígenas del centro norte de México. Investigação qualitativa em saúde, Lisboa, v. 2, pp. 1145-1154, 2017. Disponível em: <https://proceedings.ciaiq.org/index.php/ ciaiq2017/article/view/1451/1408>. Acesso em: 11 out. 2021. REIS, L. G.; PEPE V. L.; CAETANO, R. Safe motherhood in Brazil: the long road to the realization of a right. Physis Revista de Salud
Colectiva, Río de Janeiro, v. 21, n 3 , p. 1139-116o, 2011. Disponível em: <http://www. scielo.br/scielo.php?script=sci_arttext\&pid =So103-73312011000300020 >. Acesso em: 11 out. 2021.

SZULIK, D.; SZWARC, L. Era una bomba de tiempo: el derrotero de la morbilidad materna severa en el Área Metropolitana de Buenos Aires. Salud colectiva, Buenos Aires, v. 11, n. 4, p. 553-564, 2015. DOI: 10.18294/sc.2015.795

URREA, F. El cuerpo de las mujeres gestantes: un diálogo entre la bioética y el género. Revista Colombiana de Bioética, Bogotá, DC, v. 7, n. 1, p. 97-110, 2012. Disponível em: <https://pesquisa. bvsalud.org/portal/resource/pt/lil-750288>. Acesso em: 11 out. 2021.

\section{Contribución de los autores}

Rangel-Flores es responsable de conseguir el financiamiento para la investigación, construyó el protocolo, recolectó y analizó la información, elaboró la propuesta inicial y final del artículo. Rincón-Zúñiga colaboró en la construcción del protocolo, recolectó y analizó la información.

Hernández-Ibarra asesoró en los aspectos metodológicos de la investigación.

Recibido: 31/10/2019

Re-presentado: 31/10/2019

Aprobado: 27/09/2021 